\title{
Biomechanical analysis of four augmented fixations of plate osteosynthesis for comminuted mid-shaft clavicle fracture: A finite element approach
}

\author{
MING NI ${ }^{1,2^{*}}$, FANGFANG ZHANG ${ }^{3 *}$, JIONG MEI $^{3}$, CHIA-YING JAMES LIN ${ }^{2}$, \\ STACEY M.S. GRUBER ${ }^{2}$, WENXIN NIU ${ }^{4}$, DUO WAI-CHI WONG ${ }^{5,6}$ and MING ZHANG ${ }^{5,6}$ \\ ${ }^{1}$ Department of Orthopaedics, Pudong New Area Peoples' Hospital Affiliated to Shanghai University of \\ Medicine and Health Sciences, Shanghai 201299, P.R. China; ${ }^{2}$ Department of Biomedical Engineering, \\ College of Engineering and Applied Sciences, University of Cincinnati, Cincinnati, OH 45221, USA; \\ ${ }^{3}$ Department of Orthopaedics, Shanghai Sixth People's Hospital, Shanghai Jiao Tong University, Shanghai 200233; \\ ${ }^{4}$ Research Center of Disabled Rehabilitation Medicine, Yangzhi Rehabilitation Hospital, \\ Tongji University School of Medicine, Shanghai 200129; ${ }^{5}$ Department of Biomedical Engineering, \\ The Hong Kong Polytechnic University, Kowloon, Hong Kong, SAR 999077; ${ }^{6}$ The Hong Kong \\ Polytechnic University Shenzhen Research Institute, Shenzhen, Guangdong 518057, P.R. China
}

Received July 10, 2019; Accepted January 10, 2020

DOI: $10.3892 /$ etm.2020.8898

\begin{abstract}
Sufficient stabilization of comminuted mid-shaft clavicle fractures via plate fixation is difficult to achieve. Various augmentations, including interfragmentary screws and cerclage wiring, have been adopted to reinforce fixation stability. The present study aimed to assess the biomechanical stability of augmented plate fixations using the finite element method. First, a clavicle fracture model was created from CT data. Fixation was then induced using a locking compressive plate (LCP) with the following four augmentations: i) Double inner cerclage wirings (DICW), ii) double outer cerclage wirings (DOCW), iii) a single interfragmentary screw (SIS) and iv) double interfragmentary screws (DIS). Compressive and bending forces of $100 \mathrm{~N}$ were subsequently applied at the acromial region of the clavicle. The stress distribution, displacement and fracture micro-motions of the model were
\end{abstract}

Correspondence to: Professor Jiong Mei, Department of Orthopaedics, Shanghai Sixth People's Hospital, Shanghai Jiao Tong University, 600 Yishan Road, Xuhui, Shanghai 200233, P.R. China E-mail: meijiong@163.com

*Contributed equally

Abbreviations: FE, finite element; LCP, locking compressive plate; DICW, double inner cerclage wirings; DOCW, double outer cerclage wirings; SIS, single interfragmentary screw; DIS, double interfragmentary screws

Key words: clavicle fracture, internal fixation, plate, interfragmentary screw, cerclage wiring, biomechanics, finite element simulation assessed and compared. The DOCW resulted in the highest stress exerted on the LCP, followed by SIS, DICW and DIS. For the clavicle fracture, DICW, DOCW and SIS resulted in high stress levels. However, DIS fixation alone resulted in levels of stress that were below the yield strength of cortical bone. Displacement analysis revealed that DOCW fixation resulted in the greatest degree of displacement and fracture micro-motions, followed by SIS, DICW and DIS. The results indicated that SIS, DIS and DOCW may be used as augmentations of LCP fixation for comminuted mid-shaft clavicle fractures. However, DIS was the recommended augmentation due to it exerting the lowest stress and the highest stability compared with the other fixations. The DICW may be used to aid fracture reduction and plate placement in surgery but should be avoided for permanent fixation.

\section{Introduction}

The clavicle is one of the most commonly fractured bones in the human body, accounting for 2.6-5\% of all adult fractures and $44 \%$ of injuries to the shoulder girdle (1). Nearly $80 \%$ of clavicle fractures occur in the mid-shaft and the majority of these are displaced comminuted fractures (2). For patients with these fractures, nonunion rates following conservative treatment may be as high as $15 \%$, making them candidates for surgical intervention $(3,4)$.

Comminuted clavicle fractures with multiple fragments or cortical splits pose a challenge for stable fixation and early post-operative rehabilitation. Plate fixation is frequently recommended for simple clavicle fractures; however, its fixation strength is usually insufficient for comminuted clavicle fractures. To achieve better stability, certain augmented supports, including cerclage wirings and interfragmentary screws, are used in combination with plate fixation $(5,6)$. 
Cerclage wires may be wrapped around the clavicle fracture above and below the plate surface. Interfragmentary screws may be placed separately or passed through the plate. Compared with cerclage wirings, interfragmentary screws provide axial compressive force and achieve absolute stability $(7,8)$. Clinical studies have revealed that augmented fixations produce satisfactory surgical outcomes in patients $(6,9)$. However, there have been cases of implant failure and complications after augmented fixation (10). Therefore, the optimal augmented fixative remains controversial in biomechanical contexts.

Finite element (FE) analysis is a powerful biomechanical tool that allows for the control of various parameters, including loading forces, fracture type and implants, that would otherwise be difficult to assess in vivo or via cadaveric experiments. FE analysis in clavicle fractures has been used to study fixation stability, bone adaptation and for the optimization of implant design (11-13). The present study aimed to investigate the augmentations available for plate fixation.

The purpose of the present study was to analyze the biomechanical stability of clavicle plate fixation under the following four augmentations: i) Cerclage wirings below the plate; ii) cerclage wirings above the plate; iii) a single interfragmentary screw; and iv) double interfragmentary screws. The effects of these augmentations on fracture union and the degree of bone and implant stress, model displacement and fracture micro-motions were evaluated. The present study hypothesized that double interfragmentary screw fixation provides greater stability when compared with the other augmentations, enhancing the effect of comminuted mid-shaft clavicle fracture treatment.

\section{Materials and methods}

Models of clavicle and surgery simulation. The FE model was constructed using CT images of a healthy male volunteer (age, 45 years; weight, $60 \mathrm{~kg}$; height, $176 \mathrm{~cm}$ ). The volunteer received a CT scan at Pudong New Area Peoples' Hospital Affiliated to Shanghai University of Medicine and Health Sciences due to shoulder periarthritis in October 2015. The volunteer provided his written informed consent to the usage of his images for model reconstruction and analysis. The modeled clavicle consisted of cortical shell with a trabecular core. Isotropic elastic properties corresponding to normal bone were assigned. The elastic modulus was $1.0 \mathrm{GPa}$ for cancellous bone and $17 \mathrm{GPa}$ for cortical bone. Furthermore, the Poisson's ratio was 0.3 (13). The present study was approved by the Ethics Committee of Pudong New Area Peoples' Hospital affiliated to Shanghai University of Medicine and Health Sciences (Shanghai, China; approval no. 2016-16).

A type 15-B2 comminuted fracture was applied to the model clavicle by partitioning a butterfly segment at the mid-shaft (Fig. 1) in accordance with the AO classification (14). The length, width and height of the segment was 28,15 and $10 \mathrm{~mm}$, respectively. The intrusion distance of the fragment into the fracture complex was assumed to be $60 \%$. The dimensions were adopted from a currently unpublished clinical study, in which the CT images of 16 cases of comminuted mid-shaft clavicle fractures were collected and the basic characters of fragments were analyzed. There were 10 males and 6 females, with a mean age of 38.8 (range, 24-58) years. They received

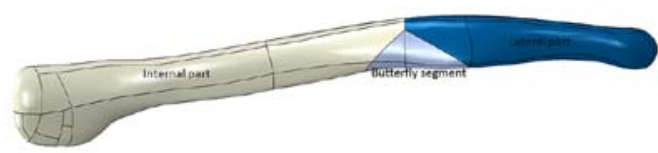

Figure 1. The Robinson type 2B1 clavicle fracture model. The clavicle was divided into three parts: Internal part (white), butterfly segment (grey) and lateral part (blue).
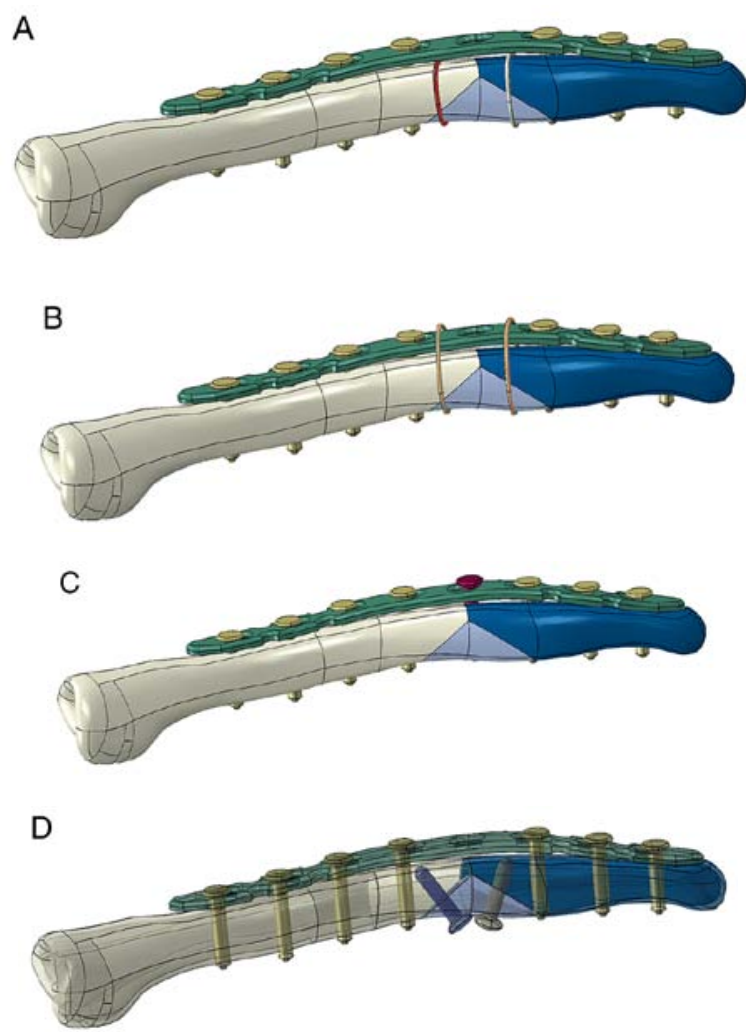

Figure 2. Geometry and position of four augmented fixations: (A) LCP with DICW; (B) LCP with DOCW; (C) LCP with SIS; and (D) LCP with DIS. LCP, locking compressive plate; DICW, double inner cerclage wirings; DOCW, double outer cerclage wirings; SIS, single interfragmentary screw; DIS, double interfragmentary screws.

standard operative treatment at Pudong New Area Peoples' Hospital Affiliated to Shanghai University of Medicine and Health Sciences between August 2015 and May 2016 and provided written informed consent for use of their data.

The implant consisted of a 3.5-mm locking compressive plate (LCP; DePuy Synthes; Johnson \& Johnson Medical $\mathrm{GmbH}$ ) containing 8 holes and was placed on the superior surface of the clavicle. The internal and lateral parts of the clavicle fracture were fixed using 4 and 3 screws, respectively. The butterfly segment was fixed using four different augmentations (Fig. 2): i) Double inner cerclage wirings (DICW), which were wrapped around the segment only; ii) double outer cerclage wirings (DOCW), which were wrapped around the segment and the LCP; iii) a single $3.5-\mathrm{mm}$ interfragmentary screw (SIS), which was placed within the screw holes of the LCP; and iv) double interfragmentary screws (DIS), which were inserted perpendicularly into the plane of the fracture. The diameter of the cerclage wirings was $1.0 \mathrm{~mm}$ and all implants were made from 316 low-vacuum-melting stainless 
Table I. Numbers of nodes and elements in the four fracture fixation models.

\begin{tabular}{lrrrr}
\hline Item & DICW & DOCW & \multicolumn{1}{c}{ SIS } & \multicolumn{1}{c}{ DIS } \\
\hline Nodes & 36,914 & 36,939 & 35,153 & 36,940 \\
Elements & 171,733 & 172,957 & 166,914 & 173,958 \\
\hline
\end{tabular}

DICW, double inner cerclage wirings; DOCW, double outer cerclage wirings; SIS, single interfragmentary screw; DIS, double interfragmentary screws.

steel with an elastic modulus of $186.4 \mathrm{GPa}$ and a Poisson's ratio of $0.3(15)$.

Boolean operations were applied to the clavicle and implants to reproduce the drilling and reaming employed during clavicle fracture surgery (13). Contact between bone and implant, and between bone fragments were considered to be frictional. The coefficient of friction for the bone-to-bone, bone-to-implant and implant-to-implant contacts were $0.46,0.42$ and 0.2 , respectively (16).

Mesh convergence. The models were meshed using 4-node linear tetrahedral element produced by Abaqus 6.13 (Dassault Systèmes SE). The global and local mesh sizes of 1 and 0.6 $\mathrm{mm}$ resulted in a $<2 \%$ deviation of stress from a coarser mesh, as demonstrated by a mesh convergence test. Therefore, the present study considered the selected mesh sizes to be acceptable. The total number of elements and nodes of the clavicle and four fixation models are presented in Table I.

Boundary and loading conditions. A total of two loading cases were applied during the present study: Cantilever bending and axial compression. A load of $100 \mathrm{~N}$ was applied in each case $(17,18)$. The forces were introduced on the acromial region, which started $15 \mathrm{~mm}$ from the lateral end. The internal end of clavicle was fixed in all degrees of freedom.

Data analysis. To calculate the model displacements along the direction of loading forces, the axis of the clavicle shaft was adjusted in line with the $\mathrm{x}$-axis of the global coordinate system. The axis of the clavicle shaft running through the midpoint of the two ends of the clavicle was judged by eye. The $y$-axis adopted the right-hand coordinate system. The $\mathrm{z}$-axis was situated in the frontal plane, vertical to the superior surface of the clavicle. Model displacement was calculated by measuring the average displacement of the acromial region. Fracture micro-motion was defined as the change of fracture gap distance after load was applied. The von Mises stress of fractures and implants of four models were also recorded and analyzed. Statistical analysis was performed in SPSS 16.0 software (SPSS Inc.) using an unpaired t-test, with $\mathrm{P}<0.05$ indicating statistically significant differences.

\section{Results}

Von Mises stress. The stress distribution and maximum von Mises stress of the four augmentations are presented in Figs. 3 and 4 and Table II. In general, stress as a result of bending was higher than that caused by compression. Under bending conditions, the DICW produced the highest stress (567.69 MPa), followed by the DOCW (281.58 MPa). The stresses applied to the DIS and SIS were 169.87 and $54.53 \mathrm{MPa}$, respectively. Under compression, the maximum stresses of DICW, DOCW, DIS and SIS were 75.34, 59.94, 21.49 and $12.39 \mathrm{MPa}$, respectively.

For the LCP, the maximum stress was concentrated at the fracture site and internal 1/3 par under two loading conditions. This was in agreement with previous biomechanical and clinical reports $(8-10,16)$ and maybe associated with the $\mathrm{S}$-shape of the plate. The stress of the LCP appeared to change when different augmentations were applied during bending. The DOCW produced the highest stress (506.64 MPa), which was 5, 47 and $31 \%$ higher than that of SIS, DIS and DICW, respectively. However, the augmentations produced similar stress to the LCP under compression.

The von Mises stress distributions of the clavicle fractures are presented in Fig. 5 and Table II. In each loading case, the DICW produced the highest stress in the clavicle, particularly at the contact area of wires and the butterfly segment. Bone stresses under DOCW and SIS fixation were relatively lower, but the internal and butterfly segments sustained considerable stress. Only with the DIS alone, the bone stresses were within the normal range of trabecular and cortical bone (19).

Fracture displacements. Acromial displacements and fracture micro-motion distances are presented in Table III. Under bending conditions, the greatest displacement was observed in the DOCW fixation $(2.59 \mathrm{~mm})$ as compared with the SIS $(2.54 \mathrm{~mm})$, DICW $(2.31 \mathrm{~mm})$ and DIS $(2.24 \mathrm{~mm}$; $\mathrm{P}<0.05)$. However, their displacements under compression were similar. The DOCW exhibited the highest micro-motions among the four supplements $(\mathrm{P}<0.05)$, while the micro-motion of SIS, DIS and DICW was similar.

\section{Discussion}

The rigid internal fixation of clavicle fractures is a necessary prerequisite for early post-rehabilitation and rapid fracture union (20). The choice of implant is critical for the treatment outcome of comminuted clavicle fractures. To observe how an adequate fixation construct should be established, an FE model of comminuted mid-shaft clavicle fracture was developed in the present study from a 45-year-old male with fixation by LCP and four augmentations. This model was selected due to being representative of the majority of typical comminuted and displaced multifragmentary fractures in adults (average age, 43 years) (9). The biomechanical performances of four augmented fixations were assessed and compared in the present study. The results revealed that the augmented fixations markedly altered the stability of clavicle fracture fixation and may implicate fracture union.

Cerclage wiring is an efficient periosteal fixation of fracture fragments for long bones, particularly the clavicle and femur $(5,21,22)$. Although the risk of periosteal vascular strangulation is a limitation that discourages surgeons to use cerclage wirings, one study has demonstrated that they have no adverse effects on cortical vascularity and produce satis- 




Figure 3. Stress distribution of four augmented fixations under two loading conditions. DICW, double inner cerclage wirings; DOCW, double outer cerclage wirings; SIS, single interfragmentary screw; DIS, double interfragmentary screws; S, Mises, von Mises stress (MPa); Avg, average.


Figure 4. Stress distribution of LCP under the (A) bending and (B) compression conditions. DICW, double inner cerclage wirings; DOCW, double outer cerclage wirings; SIS, single interfragmentary screw; DIS, double interfragmentary screws; S, Mises, von Mises stress (MPa); Avg, average.

factory clinical outcomes (23). However, the high tension and small contact area of cerclage wiring fixation may result in the local mechanical overload exceeding the strength of bone. When cerclage wirings were wrapped around the clavicle in the present study, high stresses were produced on bone and implants, exceeding the yield strength of cortical bone. This may lead to a propensity of bone necrosis and re-fracture. In addition, this result was consistent with one previous clinical study (5). However, if cerclage wirings are placed around the LCP, the plate may act as a support and the stress placed upon 
Table II. Maximum von Mises stress $(\mathrm{MPa})$ of the clavicle fracture model and implants under the four augmentations.

\begin{tabular}{|c|c|c|c|c|c|c|c|c|}
\hline \multirow[b]{2}{*}{$\begin{array}{l}\text { Fixation } \\
\text { method }\end{array}$} & \multirow[b]{2}{*}{$\begin{array}{l}\text { Loading } \\
\text { condition }\end{array}$} & \multicolumn{3}{|c|}{ Clavicle } & \multicolumn{4}{|c|}{ Implant } \\
\hline & & $\begin{array}{l}\text { Internal } \\
\text { part }\end{array}$ & $\begin{array}{l}\text { Butterfly } \\
\text { segment }\end{array}$ & $\begin{array}{l}\text { Lateral } \\
\text { part }\end{array}$ & Plate & $\begin{array}{l}\text { Interfragmentary } \\
\text { screw }\end{array}$ & $\begin{array}{l}\text { Internal } \\
\text { ring/screw }\end{array}$ & $\begin{array}{c}\text { Lateral } \\
\text { ring/screw }\end{array}$ \\
\hline \multirow[t]{2}{*}{ DICW } & Bending & 141.52 & 523.20 & 450.60 & 348.28 & - & 567.69 & 186.43 \\
\hline & Compression & 31.25 & 117.59 & 153.15 & 118.16 & - & 75.34 & 37.33 \\
\hline \multirow[t]{2}{*}{ DOCW } & Bending & 115.59 & 192.15 & 74.04 & 506.64 & - & 262.67 & 281.58 \\
\hline & Compression & 16.53 & 48.57 & 12.33 & 118.19 & - & 52.03 & 59.94 \\
\hline \multirow[t]{2}{*}{ SIS } & Bending & 181.90 & 35.93 & 98.85 & 480.75 & 54.53 & - & - \\
\hline & Compression & 32.02 & 4.81 & 13.18 & 117.99 & 12.39 & - & - \\
\hline \multirow[t]{2}{*}{ DIS } & Bending & 107.31 & 31.92 & 26.02 & 269.40 & - & 169.87 & 74.25 \\
\hline & Compression & 16.43 & 6.21 & 6.09 & 118.21 & - & 19.66 & 21.49 \\
\hline
\end{tabular}

DICW, double inner cerclage wirings; DOCW, double outer cerclage wirings; SIS, single interfragmentary screw; DIS, double interfragmentary screws. The tension and compression yield stress of trabecular was $84.9 \pm 11.2 \mathrm{MPa}$ and $135.3 \pm 34.3 \mathrm{MPa}$. The yield stress of cortical bone was on average $20-30 \%$ higher than trabecular tissue (20).

Table III. Model displacements and fracture micro-motions (mm) under the four fixations.

\begin{tabular}{llcc}
\hline $\begin{array}{l}\text { Fixation } \\
\text { method }\end{array}$ & $\begin{array}{l}\text { Loading } \\
\text { condition }\end{array}$ & $\begin{array}{c}\text { Acromial } \\
\text { displacement }\end{array}$ & $\begin{array}{c}\text { Fracture } \\
\text { micro-motion }\end{array}$ \\
\hline DICW & Bending & $2.31^{\mathrm{a}}$ & 0.17 \\
& Compression & 0.41 & 0.04 \\
DOCW & Bending & $2.59^{\mathrm{a}}$ & $0.24^{\mathrm{a}}$ \\
& Compression & 0.43 & $0.12^{\mathrm{a}}$ \\
SIS & Bending & $2.54^{\mathrm{a}}$ & 0.17 \\
& Compression & 0.42 & 0.04 \\
DIS & Bending & $2.24^{\mathrm{a}}$ & 0.15 \\
& Compression & 0.42 & 0.04 \\
\hline
\end{tabular}

${ }^{\mathrm{a}} \mathrm{P}<0.05$. DICW, double inner cerclage wirings; DOCW, double outer cerclage wirings; SIS, single interfragmentary screw; DIS, double interfragmentary screws.

bone tissue would markedly decrease. This was supported by a study by Chen et al (6), which demonstrated that 21 acute clavicle fractures treated with DOCW fixations all healed uneventfully and without complication.

Among the two cerclage wiring fixations, the results of the present study indicated that the biomechanical stability of DICW was greater than that of DOCW. This may be explained by the 'loose-lock stability' mechanism (21). 'Loose-lock stability' maybe divided into two distinct phases: The loose-displace phase and the lock-stable phase. In the first phase, fragments are allowed to displace freely. However, with the increasing displacement of fracture fragments, the wiring is tensioned and the segments are subsequently stabilized, despite a certain degree of malalignment. The DOCW may therefore exhibit larger fracture micro-motions, as the cerclage would require to accommodate more room between the plate and the clavicle.

Interfragmentary lag screw fixation is another augmentation of plate osteosynthesis for comminuted clavicle fractures. Depending on the size and geometry of fragments, one or more screws maybe used to hold them. Biomechanical studies have revealed that lag screw configurations are stiffer compared with cerclage wiring or cable systems $(7,8)$, which was also supported by the FE results of the present study. Acromial displacements of interfragmentary screw fixation were also smaller than those of cerclage wirings. In clinical practice, lag screw fixations exhibit more promising treatment results than cerclage wirings $(3,5)$. The results of the present study indicated that the DIS should be preferably recommended over the SIS in terms of lower stress magnitudes and better stability. This is compatible with the aforementioned hypothesis.

The major surgical disadvantage of DIS is its double drilling on the butterfly segment. Such a maneuver may induce the risk of segment breakage and vascular damage. In view of this, single lag screws are used. However, this compromises fixation strength and a combination of lag screw, Kirshner pin, cerclage wiring or plates may be required for the butterfly segment of a clavicle fracture (24).

The position and orientation of the implant may change the biomechanical environment of the fracture site. For the interfragmentary lag screw, it may be placed either perpendicular or oblique to the fracture plane. In most cases, the perpendicular position is simple to place and provides nearly optimal function (25). The present study indicated that the DIS was more stable than the SIS, despite the SIS providing more torque than the DIS. In addition, the screw of the DIS was collinear to the principal stress direction under bending, which may reinforce the strength of the fracture site. The results indicated that, whether placed using the plate hole or independently, the lag screw should be directed perpendicular to the fracture plane.

The FE results of the present study demonstrated that the augmented fixations may provide different construct stabilities under different loading conditions. Under compression, stress levels and acromial displacements were similar. However, when bending, these levels varied greatly. This indicated that 

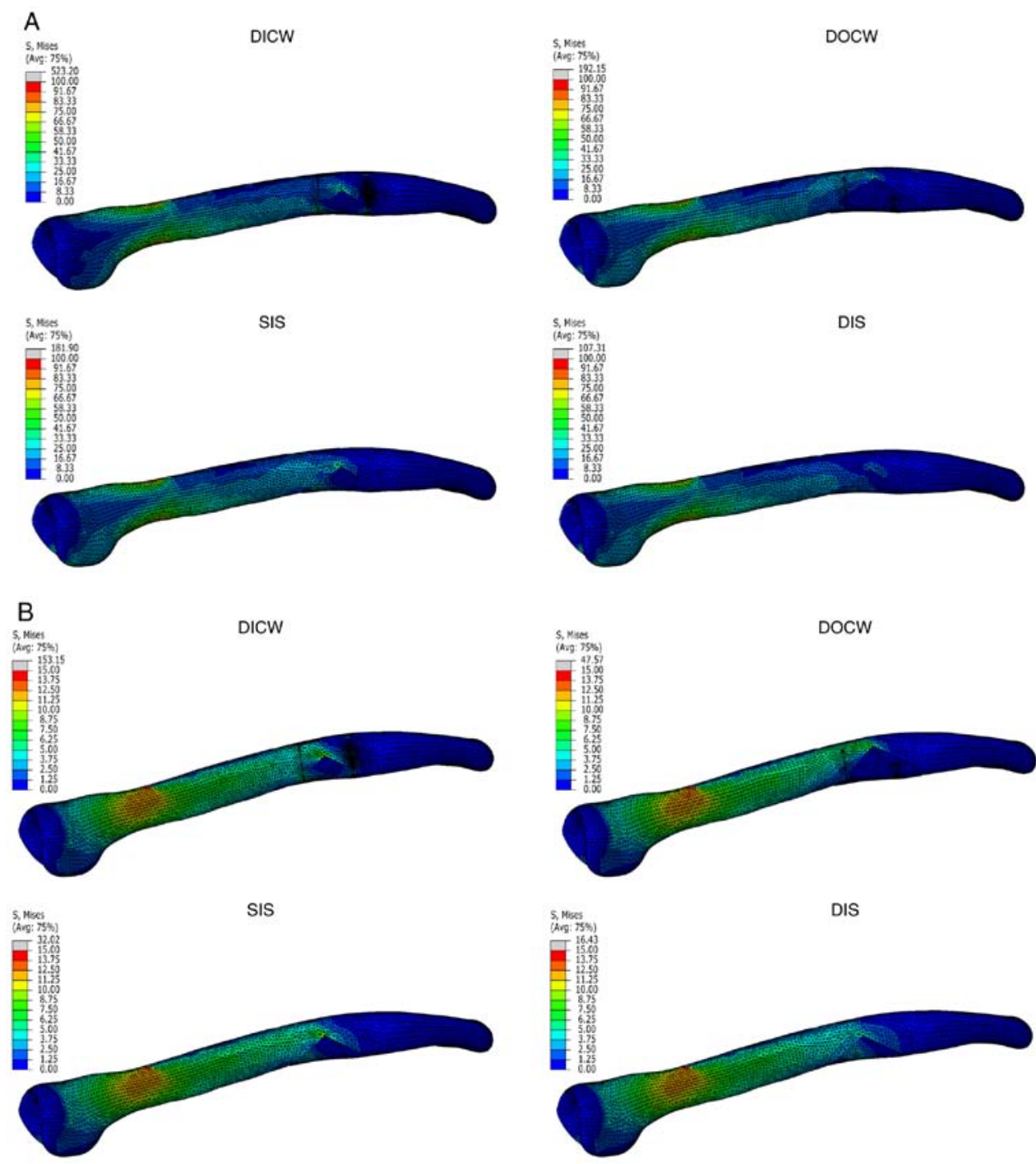

Figure 5. Stress distribution of clavicle fractures under the (A) bending and (B) compression conditions. DICW, double inner cerclage wirings; DOCW, double outer cerclage wirings; SIS, single interfragmentary screw; DIS, double interfragmentary screws; S Mises, von Mises stress (MPa); Avg, average.

augmented fixation may not effectively resist the bending force. From biomechanical and anatomical perspectives, the major bending force exerted on the clavicle originates from the gravity applied to the affected upper limb. This suggests that suspension of the affected limb after surgery is required. In addition, the weight-bearing rehabilitation of the affected limb, or any practice which may apply bending forces to the clavicle, should be avoided.

The fracture micro-motion is not only a key parameter of fixation stability, but also reflects the quality and quantity of callus formations and fracture healing. Fracture micro-motions between 0.15 and $1.0 \mathrm{~mm}$ have been determined to be optimal for bone union, and hence, a certain amount of micro-motion is required for healing (26). The present study revealed that the fracture micro-motions in all conditions were approximately within the suggested range, indicating that bone regeneration or healing would have resulted.

Despite the explicit qualitative and quantitative results obtained, the present study has a clear limitation in that no experimental test was performed to validate the results of FE. However, it should be noted that the aim was to investigate the clinical trends rather than absolute values. In this respect, the lack of experimental validation is a justified limitation. In addition, the reliability of the calculated results was further guaranteed by the use of an FE model, which was developed using one that was previously validated (10). Furthermore, convergence tests were carefully performed to guarantee the precision of FE analysis.

In conclusion, the SIS, DIS and DOCW may be used as augmentations of LCP fixation for comminuted mid-shaft clavicle fractures. The DIS featured improved fixation stability and lower stress concentration when compared with those of other methods and should be recommended for comminuted mid-shaft clavicle fracture. The DICW may also be used to aid fracture reduction and plate placement during surgery but should be avoided as a permanent fixation.

\section{Acknowledgements}

We are grateful to Dr Kuan Wang from the Rehabilitation Department of Yangzhi Rehabilitation Hospital, Tongji University School of Medicine (Shanghai, China) for his help and advice. 


\section{Funding}

This study was supported by the general project of Shanghai Municipal Health Bureau (grant no. 201840137).

\section{Availability of data and materials}

The datasets used and/or analyzed during the current study are available from the corresponding author on reasonable request.

\section{Authors' contributions}

MN, JM, WXN and FFZ designed the study and wrote the first draft of the manuscript. SMSG and FFZ collected the data. DWCW was responsible for finite element modeling. CYJL and $\mathrm{MZ}$ participated in data analysis and interpretation and revision of the manuscript. All authors have read and approved the final version of the paper.

\section{Ethics approval and consent to participate}

This study was approved by the Ethics Committee of Pudong New Area Peoples' Hospital affiliated to Shanghai Health University (approval no. 2016-16; Shanghai, China).

\section{Consent for publication}

The volunteer provided written informed consent for the publication of his digital images.

\section{Competing interests}

The authors declare that they have no competing interests.

\section{References}

1. Postacchini F, Gumina S, De Santis P and Albo F: Epidemiology of clavicle fractures. J Shoulder Elbow Surg 11: 452-456, 2002.

2. Kulshrestha V, Roy T and Audige L: Operative versus nonoperative management of displaced midshaft clavicle fractures: A prospective cohort study. J Orthop Trauma 25: 31-38, 2011.

3. Shen WJ, Liu TJ and Shen YS: Plate fixation of fresh displaced midshaft clavicle fractures. Injury 30: 497-500, 1999.

4. Canadian Orthopaedic Trauma Society: Nonoperative treatment compared with plate fixation of displaced midshaft clavicular fractures. A multicenter, randomized clinical trial. J Bone Joint Surg Am 89: 1-10, 2007.

5. Shin SJ, Do NH and Jang KY: Risk factors for postoperative complications of displaced clavicular midshaft fractures J Trauma Acute Care Surg 72: 1046-1050, 2012.

6. Chen $\mathrm{CH}$, Chen JC, Wang C, Tien YC, Chang JK and Hung SH: Semitubular plates for acutely displaced midclavicular fractures: A retrospective study of 111 patients followed for 2.5 to 6 years. J Orthop Trauma 22: 463-466, 2008.

7. Faraj AA, Naraen A and Twigg P: A comparative study of wire fixation and screw fixation in arthrodesis for the correction of hallux rigidus using an in vitro biomechanical model. Foot Ankle Int 28: 89-91, 2007.
8. Matloub HS, Jensen PL, Sanger JR, Grunert BK and Yousif NJ: Spiral fracture fixation techniques. A biomechanical study. J Hand Surg [Br] 18: 515-519, 1993.

9. Smekal V, Oberladstaetter J, Struve P and Krappinger D: Shaft fractures of the clavicle: Current concepts. Arch Orthop Trauma Surg 129: 807-815, 2009.

10. Wijdicks FJ, Van der Meijden OA, Millett PJ, Verleisdonk EJ and Houwert RM: Systematic review of the complications of plate fixation of clavicle fractures. Arch Orthop Trauma Surg 132: 617-625, 2012.

11. Favre P, Kloen P, Helfet DL and Werner CM: Superior versus anteroinferior plating of the clavicle: A finite element study. J Orthop Trauma 25: 661-665, 2011.

12. Huang TL, Chen WC, Lin KJ, Tsai CL, Lin KP and Wei HW: Conceptual finite element study for comparison among superior, anterior, and spiral clavicle plate fixations for midshaft clavicle fracture. Med Eng Phys 38: 1070-1075, 2016.

13. Ni M, Niu W, Wong DW, Zeng W, Mei J and Zhang M: Finite element analysis of locking plate and two types of intramedullary nails for treating mid-shaft clavicle fractures. Injury 47 : 1618-1623, 2016

14. Marsh JL, Slongo TF, Agel J, Broderick JS, Creevey W, DeCoster TA, Prokuski L, Sirkin MS, Ziran B, Henley B, et al: Fracture and dislocation classification compendium - 2007: Orthopaedic Trauma Association classification, database and outcomes committee. J Orthop Trauma 21: S1-S133, 2007.

15. Cronskär M, Rasmussen J and Tinnsten M: Combined finite element and multibody musculoskeletal investigation of a fractured clavicle with reconstruction plate. Comput Methods Biomech Biomed Engin 18: 740-748, 2015.

16. Goffin JM, Pankaj P and Simpson AH: The importance of lag screw position for the stabilization of trochanteric fractures with a sliding hip screw: A subject-specific finite element study. J Orthop Res 31: 596-600, 2013.

17. Iannolo M, Werner FW, Sutton LG, Serell SM and VanValkenburg SM: Forces across the middle of the intact clavicle during shoulder motion. J Shoulder Elbow Surg 19: 1013-1017, 2010.

18. Scepi M, Faure JP, Ridoux N, Kamina P and Richer JP: A three-dimensional model of the shoulder girdle. Forces developed in deltoid and supraspinatus muscles during abduction. Surg Radiol Anat 26: 290-296, 2004.

19. Bayraktar HH, Morgan EF, Niebur GL, Morris GE, Wong EK and Keaveny TM: Comparison of the elastic and yield properties of human femoral trabecular and cortical bone tissue. J Biomech 37: 27-35, 2004.

20. Asadollahi S, Hau RC, Page RS, Richardson M and Edwards ER: Complications associated with operative fixation of acute midshaft clavicle fractures. Injury 47: 1248-1252, 2016.

21. Perren SM, Fernandez Dell'Oca A, Lenz M and Windolf M: Cerclage, evolution and potential of a Cinderella technology. An overview with reference to periprosthetic fractures. Acta Chir Orthop Traumatol Cech 78: 190-199, 2011.

22. Lenz M, Lehmann W and Wähnert D: Periprosthetic fracture fixation in osteoporotic bone. Injury 47 (Suppl 2): S44-S50, 2016.

23. Kirby BM and Wilson JW: Effect of circumferential bands on cortical vascularity and viability. J Orthop Res 9: 174-179, 1991.

24. Misaghi A, Doan J, Bastrom T and Pennock AT: Biomechanical Evaluation of Plate Versus Lag Screw Only Fixation of Distal Fibula Fractures. J Foot Ankle Surg 54: 896-899, 2015.

25. Baumgart FW, Cordey J, Morikawa K, Perren SM, Rahn BA, Schavan R and Snyder S: AO/ASIF self-tapping screws (STS). Injury 24 (Suppl 1): S1-S17, 1993.

26. Jagodzinski M and Krettek C: Effect of mechanical stability on fracture healing - an update. Injury 38 (Suppl 1): S3-S10, 2007.

This work is licensed under a Creative Commons Attribution-NonCommercial-NoDerivatives 4.0 International (CC BY-NC-ND 4.0) License. 